Sammlung Metzler Band 299 
Gerald A. Fetz

\section{Martin Walser}

Verlag J.B. Metzler

Stuttgart - Weimar 
Die Deutsche Bibliothek - CIP-Einheitsaufnahme

Fetz, Gerald A.:

Martin Walser / Gerald A. Fetz. -

- Stuttgart ; Weimar : Metzler, 1997

(Sammlung Metzler ; Bd. 299)

ISBN 978-3-476-10299-7

NE: GT

ISBN 978-3-476-10299-7

ISBN 978-3-476-03995-8 (eBook)

DOI 10.1007/978-3-476-03995-8

ISSN 0558-3667

SM 299

Dieses Werk einschließlich aller seiner Teile ist urheberrechtlich geschützt. Jede Verwertung außerhalb der engen Grenzen des Urheberrechtsgesetzes ist ohne Zustimmung des Verlages unzulässig und strafbar. Das gilt insbesondere für Vervielfältigungen, Übersetzungen, Mikroverfilmungen und die Einspeicherung und Verarbeitung in elektronischen Systemen.

(C) 1997 Springer-Verlag GmbH Deutschland

Ursprünglich erschienen bei J.B. Metzlersche Verlagsbuchhandlung und Carl Ernst Poeschel Verlag GmbH in Stuttgart 1997 


\section{Inhalt}

Vorbemerkung

1. „Von Wasserburg an" - Zum Leben Martin Walsers .... 1

2. Walsers Anfang als Schriftsteller .................................... 12

2.1 Hörspiele und weitere Arbeiten fürs Radio .................. 13

2.2 Beschreibung einer Form. Walsers Doktorarbeit zu Kafka ................................................................ 16

2.3 Ein Flugzeug über dem Haus und andere Geschichten ... 18

3. Die ersten Romane ...................................................... 28

3.1 Ehen in Philippsburg.................................................. 28

3.2 Die Anselm-Kristlein-Trilogie ......................................... 39

3.2.1 Halbzeit ........................................................... 39

3.2.2 Das Einhorn …………………………………... 53

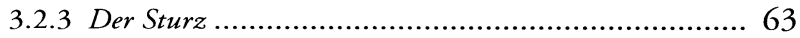

4. Die Theaterstücke der 60er Jahre …………………....... 73

4.1 Der Abstecher ............................................................ 77

4.2 Eiche und Angora ......................................................... 80

4.3 Überlebensgroß Herr Krott ........................................... 87

4.4 Der schwarze Schwan .................................................. 89

4.5 Die Zimmerschlacht ...................................................... 95

4.6 Ein Kinderspiel .............................................................. 99

5. Prosa nach der Kristlein-Trilogie ................................... 104

5.1 Die Gallistl'sche Krankheit ......................................... 105

5.2 Die Franz-Horn-Romane ............................................ 110

5.2.1 Jenseits der Liebe ................................................. 110

5.2.2 Der Brief an Lord Liszt ....................................... 114

5.3 Die Helmut-Halm-Romane ...................................... 118

5.3.1 Ein fliehendes Pferd .......................................... 118

5.3.2 Brandung ............................................................... 123

5.4 Die Zürn-Romane ................................................. 129

5.4.1 Seelenarbeit .......................................................... 129

5.4.2 Das Schwanenhaus .............................................. 134

5.4.3 Jagd ……........................................................... 137 
5.5 Die - vorläufig - letzten Romane

5.5.1 Die Verteidigung der Kindheit ............................. 139

5.5.2 Ohne einander ............................................... 145

5.5.3 Finks Krieg .................................................. 151

6. Die Künstler-Dramen ............................................. 155

6.1 Das Sauspiel ..................................................... 155

6.2 In Goethes Hand .................................................. 162

6.3 Die Ohrfeige ........................................................ 168

7. Walser und Deutschland .......................................... 171

7.1 Dorle und Wolf ........................................................ 178

7.2 Über Deutschland reden ........................................ 182

7.3 Nach dem Fall der Mauer ...................................... 185

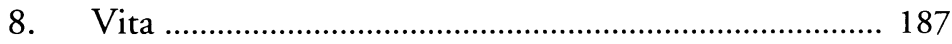

9. Bibliographie ................................................... 190

9.1 Werkverzeichnis ................................................... 191

9.2 Sekundärliteratur: Eine Auswahl ........................... 197

9.3 Interviews: Eine Auswahl ................................... 216 


\section{Vorbemerkung}

Es ist das Hauptziel dieses Buches, eine kritische Einführung in das bisherige Gesamtwerk des bundesdeutschen Schriftstellers Martin Walser anzubieten. Zweifellos gilt Walser heute - neben wenig anderen - als einer der anerkanntesten und wichtigsten deutschsprachigen Autoren der Nachkriegszeit, unter dessen beinahe zahllosen Texten Hauptwerke fast aller zeitgenössischen literarischen Gattungen zu finden sind. Da er und sein Werk teilweise heftig umstritten sind, ist es angebracht, im Rahmen der Diskussionen zu den einzelnen Werken und Gattungen, einen Einblick in die noch immer stark wachsende Sekundärliteratur zu geben. In diesem Zusammenhang beschäftigen wir uns mit der recht unterschiedlichen deutschund englischsprachigen Rezeption zum Leben und Werk Walsers.

Walser ist ein umstrittener Autor, weil er sich immer wieder in öffentliche Diskussionen einmischt und weil er häufig in Reden und Veröffentlichungen unangenehme politische und gesellschaftliche Themen aufgreift und provozierende Standpunkte vertritt. Seit den frühesten Werken ist Walser ein moralischer, ein engagierter und für viele Kritiker - und manche Leser - ein unangenehmer Schriftsteller. Es scheint uns also unumgänglich, den politischen und historischen Kontext der Werke und Reden in die jeweilige Diskussion einzubeziehen.

Es wird hier nicht angestrebt, eine alle Rätsel lösende Interpretation des Walserschen Gesamtwerks anzubieten, aber Analysen zu den größeren Einzelwerken sowie zu den verschiedenen literarischen Formen werden gebracht. In Anerkennung seiner Bedeutung als "Mitarbeiter an der öffentlichen Meinung" bekam Walser 1994 den neu gestifteten Dolf-Sternberger-Preis für öffentliche Rede. Aus Platzmangel können wir Walsers Aufsätzen und Reden kein eigenes Kapitel widmen, aber es würde sich zweifellos lohnen, diese Schriften und Aussagen, die in mindestens dreizehn verschiedenen Sammlungen zusammengebracht sind, ausführlich zu untersuchen. In der Diskussion der Kritik beschränken wir uns im folgenden fast ausschließlich auf die deutsch- und englischsprachige Sekundärliteratur. Untersuchungen zur Walser-Kritik in weiteren Sprachbereichen dem französischen, italienischen oder russischen etwa - könnten jedoch einen wichtigen Beitrag zum Verständnis seiner Wirkung über den deutschsprachigen Raum hinaus leisten. Diesbezüglich würde es 
ebenfalls interessant sein, die Gründe dafür zu untersuchen, warum Walsers Werke, trotz sehr guter Übersetzungen ins Englische, sehr wenig anhaltenden Nachhall im englischsprachigen Raum aufweisen können: zur Zeit sind in den USA alle Werke Walsers in englischer Übersetzung außer dem Roman Das Einhorn vergriffen.

Dieses Buch wäre ohne die vielen Anregungen und Einsichten nicht möglich gewesen, die ich durch die ,Vorarbeiten zahlreicher Literaturkritiker und -wissenschaftler gewonnen habe. In dieser Hinsicht sind die Untersuchungen der renommiertesten ,WalserSpezialisten` Thomas Beckermann, Klaus Pezold, Heike Doane, Anthony Waine und Alexander Mathäs besonders hervorzuheben. Von ihnen - und anderen - habe ich über Walser und seine Werke sehr viel gelernt. An dieser Stelle möchte ich auch Herrn und Frau Walser für ihre Gastfreundschaft während eines ganztägigen Besuches bei ihnen in Überlingen danken, sowie Herrn Walser für seine Bereitschaft, meine Fragen mit großer Geduld zu beantworten und mit mir über Literatur und Politik, über mein Land und seines zu reden. Mein Dank gilt ebenfalls meinen zwei wichtigsten ,Germanistiklehrern`, meinem Doktorvater Professor Peter Gontrum an der Universität Oregon/USA und meinem vorbildlichen Kollegen an der Universität Montana/USA, Professor Horst Jarka. Einigen weiteren Freunden und Kollegen - Dennis McCormick, Karlheinz Finken, Elizabeth Ametsbichler, James Reece und Erich Pohl - möchte ich auch für ihre Bereitschaft danken, Teile meines Manuskriptes zu lesen und mit mir darüber zu diskutieren. Von ihnen allen habe ich Ermutigung, Anregungen und Einsichten bekommen. Mein besonderer Dank gilt auch meiner Lektorin beim Metzler Verlag, Frau Ute Hechtfischer, die mir zahllose Verbesserungsvorschläge gemacht und dabei sehr viel Geduld gezeigt hat. Viele der Stärken dieses Buches, falls es welche gibt, sind auf sie zurückzuführen; für die Schwächen kann ich sie aber leider nicht verantwortlich machen: sie konnte gewiß nicht alle beseitigen.

Die Forschung für dieses Buch wurde z.T. durch die finanzielle Unterstützung der Universität Montana und der Fulbright-Kommission ermöglicht. Ihnen und ihren zuständigen Vertretern gebührt gleichfalls mein Dank.

Dieses Buch möchte ich meiner Frau Sallie Scott und unseren Kindern, Christian, Andreas und Gillian, sowie dem Andenken meiner Mutter, Ruth Krug Fetz, widmen. 